Cavendish-HEP-95/07

hep-ph/9601220

January 1996

\title{
OEDIPUS: Onium Evolution, Dipole Interaction and Perturbative Unitarisation Simulation
}

\author{
G.P. Salam \\ Cavendish Laboratory, Cambridge University, \\ Madingley Road, Cambridge CBз OHE, UK \\ e-mail: salam@hep.phy.cam.ac.uk
}

\begin{abstract}
OEDIPUS is a Monte Carlo simulation program which can be used to determine the small- $x$ evolution of a heavy onium using Mueller's colour dipole formulation, giving the full distribution of dipoles in rapidity and impact parameter. Routines are also provided which calculate onium-onium scattering amplitudes between individual pairs of onium configurations, making it possible to establish the contribution of multiple pomeron exchange terms to onium-onium scattering (the unitarisation corrections).
\end{abstract}

${ }^{*}$ Research supported by the UK Particle Physics and Astronomy Research Council 


\section{PROGRAM SUMMARY}

Title of program: OEDIPUS

Catalogue number:

Program obtainable from: ftp://axpf.hep.phy.cam.ac.uk/pub/theory/oedipus.tar.gz , see also http://www.hep.phy.cam.ac.uk/theory/software/oedipus.html

Licensing provisions: None

Computers: Tested on Dec Alpha, Sun

Operating system: Unix (OSF3.2, SunOS-4.1.3, Solaris-2.4)

Program language used: Fortran-90

Memory required to execute with typical data: 1-10 MB

No. of bits in a word: $32 / 64$

No. of lines in distributed program, including test data, etc.: 6186

Keywords: Small-x; BFKL; Pomeron; Onium-onium scattering; Dipoles; Monte Carlo; Unitarity.

Nature of the physical problem

BFKL evolution [1] of a heavy (quark-)onium, to give full information on the rapidity and transverse positions of gluons carrying a small fraction $x$ of the onium's longitudinal momentum. This information can be used to calculate a variety of onium-onium scattering cross sections, including multiple pomeron contributions, which restore unitarity.

Method of solution

A Monte Carlo simulation of the evolution in rapidity of the gluon structure of the onium, using Mueller's colour dipole formulation of small- $x$ physics [2].

Restrictions on the complexity of the problem

The number of dipoles (gluons) in an onium grows as an exponential of the rapidity, and inversely with the value of a cutoff used to regulate an ultra-violet divergence. The time for evolution of an onium is proportional to the number of dipoles, while that for calculation of the onium-onium scattering amplitude goes as the square of the number of dipoles. Memory restrictions also arise from the need to store large configurations of dipoles (very large 
fluctuations can occur in the number of dipoles present).

Typical running time

On a DEC Alpha 3000 processor, evolution generates about 10000 gluons per second. At a rapidity $Y=16$ and an ultra-violet cutoff of 0.01 times the onium size, a day's running will generate about $10^{5}$ onium-onium scatterings.

References

[1] Y. Y. Balitskiǐ and L. N. Lipatov, Sov. Phys. JETP 28 (1978) 822;

E. A. Kuraev, L. N. Lipatov, and V. S. Fadin, Sov. Phys. JETP 45 (1977) 199;

L. N. Lipatov, Sov. Phys. JETP 63 (1986) 904.

[2] A. H. Mueller and B. Patel, Nucl. Phys. B 425 (1994) 471;

A. H. Mueller, Nucl. Phys. B 437 (1995) 107.

\section{LONG WRITE-UP}

\section{Introduction}

There has been much interest recently in BFKL [1, 2, 3] type processes and their possible observation at HERA and the Tevatron. Calculation of the cross sections for a number of these processes, such as diffractive dissociation (see e.g. [四, 5]) or exclusive vector meson production (e.g. [6]) requires detailed information about the dominant exchanged "object", known as the BFKL pomeron; for example one needs an understanding of the triple pomeron vertex and of the transverse distribution of small- $x$ gluons. At present, the only BFKL type process which is fully calculable in perturbative QCD is high energy onium-onium scattering. Mueller's colour dipole formulation of this process [7, 8, 9, 10] (for a related approach, see [11, 12]), offers a well defined way of performing the necessary calculations, in the large $N_{C}$ approximation, where $N_{C}$ is the number of colours.

The colour dipole formulation of small- $x$ evolution and high energy onium-onium scattering is particularly suited to Monte Carlo simulation, since the evolution is probabilistic in nature, and because each branching (of one dipole into two) is independent of all other branchings.

This paper is divided into three parts. The first gives a brief overview of the small$x$ dipole evolution of an onium and of the issues relevant to a Monte Carlo simulation of such an evolution. The second section examines the use of the dipole structures of a pair of evolved onia to calculate onium-onium scattering amplitudes, including the multiple pomeron 
exchange contributions which restore unitarity. The final section gives a detailed description of the structure and use of the OEDIPUS package.

\section{Dipole structure of an onium}

\subsection{Background}

One starts with an onium where the transverse separation between the quark and anti-quark is $\mathbf{b}_{01}$. The probability of generating a gluon at a point $\mathbf{b}_{2}$ carrying a small fraction $e^{-y}$ of the light-cone momentum of the onium is [7]:

$$
\frac{\mathrm{d} P}{\mathrm{~d} y \mathrm{~d}^{2} \mathbf{b}_{2}}=e^{-y / \lambda} \frac{\alpha_{S} N_{C}}{2 \pi^{2}} \frac{b_{01}^{2}}{b_{02}^{2} b_{12}^{2}}
$$

$N_{C}$ is the number of colours $\left(N_{C}=3\right.$ for QCD), $\alpha_{S}$ is the strong coupling constant; $\lambda$, the effective lifetime in rapidity $(y)$ of the dipole, is related to the virtual corrections (or more intuitively, conservation of probability):

$$
\lambda^{-1}=\frac{\alpha_{S} N_{C}}{2 \pi^{2}} \int \mathrm{d}^{2} \mathbf{b}_{2} \frac{b_{01}^{2}}{b_{02}^{2} b_{12}^{2}} .
$$

In the limit of large $N_{C}$, the colour structure means that the two new dipoles which arise $\left(\mathbf{b}_{02}\right.$ and $\left.\mathbf{b}_{12}\right)$ can themselves independently emit gluons, while the original colour dipole is effectively destroyed. This branching of dipoles repeats itself until the rapidity of any new gluons which would be produced exceeds the maximum rapidity available. Through this branching process, one can establish the probability of any given configuration of dipoles.

\subsection{Notes on implementation}

One of the main aspects of eq. (11) is that it has non-integrable divergences at $b_{02}=0$ and $b_{12}=0$. This leads to the integral for $\lambda^{-1}$ being infinite. A solution to this problem is to introduce a cutoff on the dipole size, eliminating any region of $\mathbf{b}_{2}$ where $b_{02}<\rho$ or $b_{12}<\rho$.

This has to be used in both eq. (11) and eq. (2). The lifetime of a dipole of size $b$ is therefore a function of $b / \rho$.

In the limit of small $\rho$ the effects of the cutoff should disappear. However the number of small dipoles is large: the number of dipoles of size $c$ from an onium of size $b$, after evolution through $y$ is approximately:

$$
n^{(1)}(c, b, y) \mathrm{d} \log c \simeq \frac{b}{c \sqrt{\pi k y}} \exp \left(\left(\alpha_{\mathcal{P}}-1\right) y-\log (c / b)^{2} / k y\right) \mathrm{d} \log c .
$$


This is valid for $|\log (c / b)| \ll k y$. The BFKL power is $\left(\alpha_{\mathcal{P}}-1\right)=4 \log 2 \alpha_{S} N_{C} / \pi$, and $k=14 \alpha_{S} N_{C} \zeta(3) / \pi$, with $\zeta(3) \simeq 1.202$ being the Riemann zeta function. The smallest dipoles will be of size $c \sim \rho$, so as $\rho$ is lowered the number of dipoles rises. In addition, the number of dipoles increases exponentially with rapidity. The time taken to generate an onium is proportional to the number of dipoles. The time to calculate onium-onium interactions is proportional to the product of the number of dipoles in the two onia.

A final complication will arise because there are very large fluctuations in the numbers of dipoles [13] - the probability of obtaining a configuration with $n$ dipoles goes as $\exp \left[-\pi(\log n)^{2} / 4 \alpha_{S} N_{C} y\right]$, so that one has to allow for configurations which are very much larger than the mean. Since configurations generally need to be stored (for example to investigate interactions between pairs of configurations), this can lead to considerable memory consumption, especially at large rapidities.

The facility of imposing an upper limit on dipole sizes during the evolution is also included, to allow a crude investigation into the uncertainties due to infra-red effects. When the implementation of the upper cutoff is turned on, both dipoles produced from a branching are required to be smaller than the upper cutoff. The lifetimes of dipoles are adjusted accordingly.

The evolution is carried out with fixed $\alpha_{S}$, which is justified in the limit of very heavy onia. There is no known unique way of including a running coupling constant, however it would be possible to modify the program to implement a scheme such as that used by Nikolaev and Zakharov [11.

\section{Onium-onium scattering}

\subsection{Background}

One wishes to obtain the amplitude $F\left(\mathbf{b}, \mathbf{b}^{\prime}, \mathbf{r}, Y\right)$, for elastic scattering between two onia of sizes (and orientations) $\mathbf{b}$ and $\mathbf{b}^{\prime}$, whose centres are separated by a transverse distance $\mathbf{r}$, with a total rapidity between them of $Y(\simeq \log s$, where $\sqrt{s}$ is the centre of mass energy).

Let $\gamma$ be a particular dipole configuration for an onium, which contains dipoles of position and size $\left(\mathbf{r}_{1}, \mathbf{c}_{1}\right) \ldots\left(\mathbf{r}_{n_{\gamma}}, \mathbf{c}_{n_{\gamma}}\right)$. The interaction between two such onia, moving in opposite directions is [8, 9, 10]:

$$
f_{\gamma, \gamma^{\prime}}=\sum_{i=1}^{n_{\gamma}} \sum_{j=1}^{n_{\gamma^{\prime}}} f\left(\mathbf{r}_{i}-\mathbf{r}_{j}^{\prime}, \mathbf{c}_{i}, \mathbf{c}_{j}^{\prime}\right),
$$

where $f\left(\mathbf{r}, \mathbf{c}_{i}, \mathbf{c}_{j}^{\prime}\right)$, the interaction between dipoles of size $\mathbf{c}$ and $\mathbf{c}^{\prime}$, whose centres are separated 
by $\mathbf{r}$ is [9, 14]:

$$
f\left(\mathbf{r}, \mathbf{c}, \mathbf{c}^{\prime}\right)=\frac{\alpha_{S}^{2}}{2}\left[\log \frac{\left|\mathbf{r}+\mathbf{c} / 2-\mathbf{c}^{\prime} / 2\right|\left|\mathbf{r}-\mathbf{c} / 2+\mathbf{c}^{\prime} / 2\right|}{\left|\mathbf{r}+\mathbf{c} / 2+\mathbf{c}^{\prime} / 2\right|\left|\mathbf{r}-\mathbf{c} / 2-\mathbf{c}^{\prime} / 2\right|}\right]^{2} .
$$

One has to average $f_{\gamma, \gamma^{\prime}}$ over dipole configurations $\gamma, \gamma^{\prime}$ of the two onia. Therefore at the level of one pomeron exchange, the elastic amplitude is:

$$
F^{(1)}\left(\mathbf{b}, \mathbf{b}^{\prime}, \mathbf{r}, Y\right)=-\sum_{\gamma, \gamma^{\prime}} P_{\gamma}\left(\mathbf{r}_{0}, \mathbf{b}, y\right) P_{\gamma^{\prime}}\left(\mathbf{r}_{0}+\mathbf{r}, \mathbf{b}^{\prime}, Y-y\right) f_{\gamma, \gamma^{\prime}}
$$

where $P_{\gamma}\left(\mathbf{r}_{0}, \mathbf{b}, y\right)$ is the probability of obtaining a dipole configuration $\gamma$ after evolution through $y$ of an onium of size $\mathbf{b}$ centred at $\mathbf{r}_{0}$. Note that in this case the result can be shown to be independent of $y$, the division of rapidity between the two onia (or equivalently of the longitudinal frame in which the calculation is performed).

One can also calculate contributions to the amplitude involving the exchange of $k$ pomerons [9]:

$$
F^{(k)}\left(\mathbf{b}, \mathbf{b}^{\prime}, \mathbf{r}, Y\right)=\frac{1}{k !} \sum_{\gamma, \gamma^{\prime}} P_{\gamma}\left(\mathbf{r}_{0}, \mathbf{b}, y\right) P_{\gamma^{\prime}}\left(\mathbf{r}_{0}+\mathbf{r}, \mathbf{b}^{\prime}, Y-y\right)\left(-f_{\gamma, \gamma^{\prime}}\right)^{k} .
$$

These multi-pomeron terms, though formally non-leading in $1 / N_{C}$, are enhanced at high rapidities by the large numbers of dipoles in each onium. All numbers of pomeron exchange can be resummed to give an amplitude $F$ which explicitly satisfies the unitarity bound:

$$
F^{(k)}\left(\mathbf{b}, \mathbf{b}^{\prime}, \mathbf{r}, Y\right)=-\frac{1}{k !} \sum_{\gamma, \gamma^{\prime}} P_{\gamma}\left(\mathbf{r}_{0}, \mathbf{b}, y\right) P_{\gamma^{\prime}}\left(\mathbf{r}_{0}+\mathbf{r}, \mathbf{b}^{\prime}, Y-y\right)\left(1-\mathrm{e}^{-f_{\gamma, \gamma^{\prime}}}\right) .
$$

These last two equations are approximations based on there being a large number of dipoles interacting. Multiple pomeron contributions should be calculated in the frame $y=Y / 2$, where corrections that would arise from wave-function saturation, which is not being calculated, are expected to be smallest [9] (see also [15]).

\subsection{Implementation considerations}

One of the main difficulties in calculating onium-onium scattering amplitudes is the large numbers of interactions which have to be worked out: in principle the product of the numbers of dipoles in each onium, multiplied by the number of points at which one needs to know the interaction.

The first way to ease the problem is to note that the dipole-dipole interaction, eq. (5), is relatively local: at large distances it dies off as $1 / r^{4}$ where $r$ is the separation between the 
centres of the dipoles. Therefore it should be safe to neglect the interaction between dipoles if their separation is sufficiently large, say greater than twice the sum of their sizes. This is found to reproduce the total dipole-dipole interaction to better than one percent!.

One generally wants to know the amplitude at many different impact parameters (i.e. onium-onium separations), for example to work out the total cross section (with the normalisation used above, just twice the amplitude, integrated over all impact parameters). One way to do this is to work out the interaction on a grid. Since there are large fluctuations in the spatial extent of the dipole configurations, the grid size has to be variable - it should completely cover the area where dipole-dipole interactions are significant. By summing the interactions at each point of the grid one obtains an approximation to the total amplitude for that configuration pair (the finer the grid, the better the approximation).

This approach is necessary if one wants to find a good approximation to the total amplitude for each configuration pair, however it is slow and does not offer any easy way to store the results so that they can be retrieved for later analysis (say if one only wanted to look at 1 and 2 pomeron exchange, but after the run, decided that 3 pomeron exchange would also be interesting) - if one uses a grid which is $100 \times 100$, then one requires 40 kilobytes per pair of configurations, for a single value of rapidity. To gain adequate accuracy one needs several tens of thousands of events; with, say, 5 values of rapidity this gives several gigabytes of data.

One reason why it is important to sample a large number of configuration pairs, is that rare, large configurations contribute significantly to the total cross section [14]. Multiple pomeron contributions are dominated by rare, dense configurations. For a given pair of dipole configurations, however, the variation of the amplitude (at those points where it is significant) with impact parameter is not so large. So it can be advantageous to evaluate fewer points in impact parameter (i.e. making a worse approximation to the amplitudes of individual configuration pairs) in exchange for a larger sample of configurations. The limit of this is to evaluate the onium-onium interaction, not on a grid, but along a radial line. In addition, because there are fewer points in impact parameter it becomes tractable to store the results. In fact the individual amplitudes are not stored event by event, rather a probability distribution for the amplitude is stored at consecutive bins in radial position. This information can then be retrieved and used to determine whichever quantity one is interested in.

\footnotetext{
${ }^{1}$ Note that this may not correctly reproduce the profile of the interaction at very large onium-onium separations, where the density of dipoles is also dying off also as $1 / r^{4}$ (though enhanced by double leading logarithmic factors). However this region is not important for the total interaction.
} 


\section{Structure and use of OEDIPUS}

The program comes in many different files in various directories: basic_src/ contains all the common subroutines which are involved with data input and output, initialisation and evolution of onia, as well as routines for determining onium-onium interaction amplitudes. It does not contain any code for analysing the results from the evolution or interaction: this must be provided as a "driver" program by the user. A number of examples are to be found in the directory samples/, including drivers to examine the spatial distribution of dipoles in the onium wave function and to determine the total and elastic scattering cross sections.

\subsection{Storage of gluons and dipoles and evolution}

Gluons and dipoles each have a specific data type:

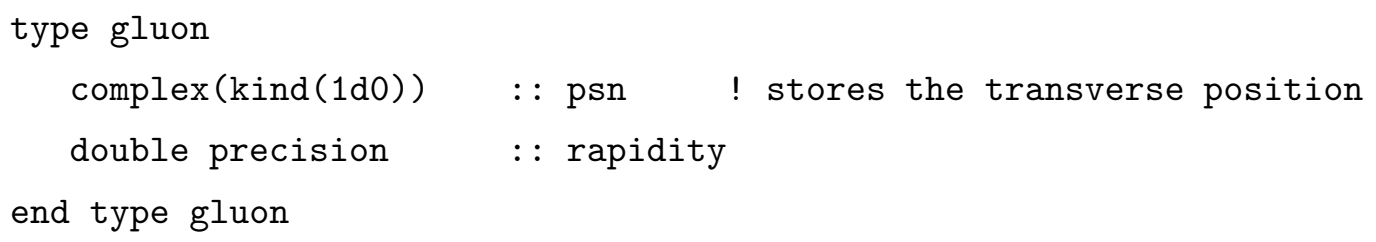

Complex variables are used throughout the program to deal with transverse positions. The dipole type is

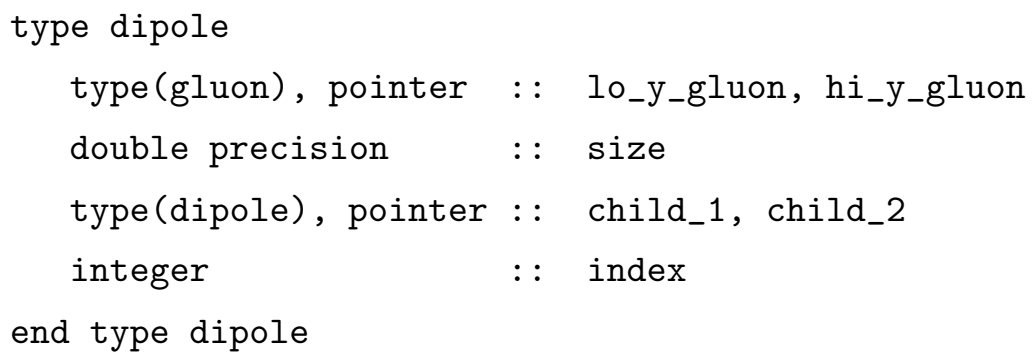

It is useful to separate the gluon content of the dipole into high rapidity and low rapidity, mainly for ease of coding. In the case of the initial onium, the two quarks are represented by gluon types. The child_1 and child_2 pointers are needed to store the tree structure of the evolved onium. If they are not associated then the dipole has no descendents (for example, because any descendents would have a rapidity larger than the maximum of the evolution). Each dipole in an onium has a different index — this can be useful in analysing the branching structure.

The following sequence of routines is needed to produce an onium and evolve it, for each loop (or "event") of the program. First, various internal counters must be reset by calling 
subroutine reset_counters

Then an onium is produced with

subroutine init_onium(onium, onm_size, phi)

This returns a pointer onium to a dipole of size onm_size, centred at $(0,0)$, with orientation phi. To produce a randomly oriented dipole, call

subroutine init_onium_rnd_phi(onium, onm_size)

The onium is evolved up to a rapidity maxy by calling

subroutine evolve_onium(onium, maxy, n_tree)

where, on return, n_tree is the total number of dipoles in the tree. To be able to analyse the results, one wants only the dipoles associated with a particular rapidity y, so one must extract them with:

subroutine extract_onium_dpls(onium, y, extrctd_dpls, n_extrctd)

The array extrctd_dpls $(:)$ is of type dpl_pntr (arrays of pointers are not permitted in Fortran 90 - the solution to this is to define a type which contains nothing but a pointer), and on return contains pointers to the n_extrctd dipoles. The array must be sufficiently large: one can ensure that it is, by dynamically allocating it to be of size (n_tree / $2+1)$. The array of dipole pointers can then be processed by the user.

This sequence allows one to first evolve the onium up to the maximum rapidity that one is interested in, and then examine the dipole structure over a range of rapidities.

Additional onia (for use simultaneously) can be produced, evolved, and the dipoles extracted, by repeating the same set of calls (omitting the call to reset_counters).

\subsection{Onium-onium interaction}

Two sets of routines are provided which calculate amplitudes for onium-onium scattering. The first returns a reasonable estimate for the elastic scattering amplitude between a pair of onium configurations, for all relevant impact parameters:

subroutine onm_onm_grid(ext_dpls1, n1, ext_dpls2, n2, grid, gh_lo, \&

\& gh_int, gv_lo, gv_int) 
It takes a pair of dipole sets ( $\mathrm{n} 1$ dipoles in ext_dpls1 and $\mathrm{n} 2$ dipoles in ext_dpls2) and determines the range of relative separations over which they interact. The onium-onium interaction is then determined for each point of a grid covering this area $(\operatorname{grid}(:,:)$ must be a two-dimensional square array; its size determines the resolution of the sampling) — the program returns information relating the array to the physical grid being used: the horizontal and vertical positions of the bottom left hand edge of the grid (gh_lo and gv_lo), as well as the horizontal and vertical spacing (or interval) of the grid (gh_int and gv_int). For each dipole pair, it only works out the contribution to those points on the grid where the dipoledipole separation is not too large (as discussed in section 3.2). The amplitudes are always worked out at the centres of the grid rectangles.

The second approach works out a binned probability distribution for the amplitude in many sections along a radial line. The details of the binning are held in the binning module which provides a variable bn of type bin_prms:

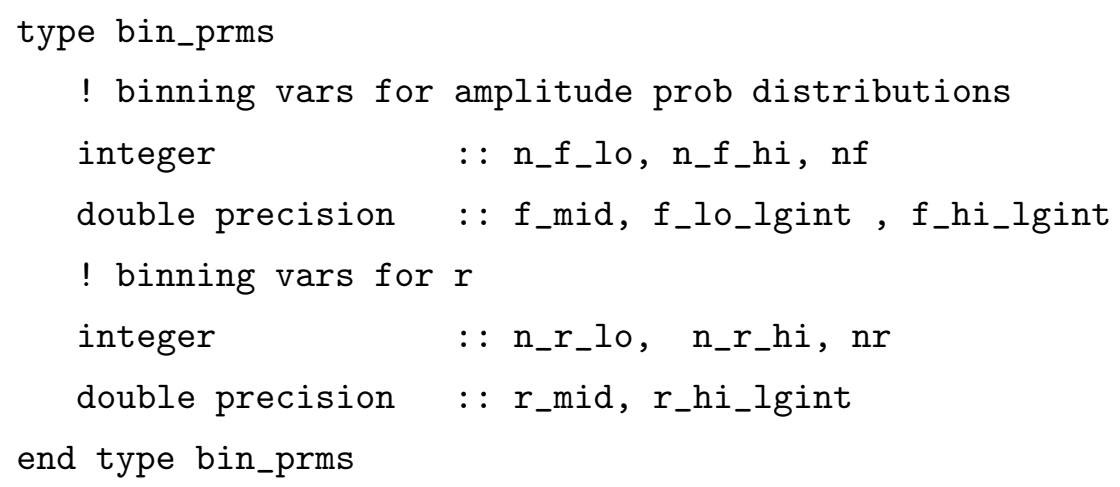

The binning of the amplitude probability distribution is divided into two regions: the lower region is for amplitudes below $f \_m i d$ and the bins are indexed 0 to ( $\left.n_{-} f_{\_} l_{0}-1\right)$, logarithmically spaced with an interval f_lo_lgint. It is important to have a bin explicitly for the absence of interaction (otherwise when integrating the interaction over a large area the smallest bin amplitude could contribute significantly) — this is bin -1 . The lowest non-zero bin must be sufficiently small to accurately reproduce the integral of the dipole-dipole interaction (bearing in mind that because the interaction dies off as $1 / r^{4}$, there are regions where the amplitude can be quite small, but whose overall contribution to the amplitude is non-negligible). It is to allow adequate coverage of this wide range of small amplitudes that logarithmic binning (in the small amplitude region) is used. For amplitudes above $f$ mid the binning is performed logarithmically with an interval $f \_$hi_lgint. The large amplitude region will contribute more significantly to the total amplitude and dividing the binning into two regions allows one to sample it more accurately in the regions which are more important. The reason to have logarithmic binning for large amplitudes is to be able to store the very large amplitudes which can 
occur at large rapidities. It is sometimes useful to have the index of the highest amplitude bin, which is $n f=n_{-} f \_l o+n_{-} f \_h i$. The variable bn is initialised with default values which reproduce total amplitudes to an accuracy of about one percent.

The binning in $r$, the distance along the radius, is also divided into two regions, but for different reasons: there are occasional events with very large transverse extents. To be able to include them with linearly spaced bins would require a prohibitively large number of bins. So for large $r$, logarithmic spacing of the bins is useful. But for small $r$, the most efficient sampling is one where each bin corresponds to a region of similar area - linear binning in $r$ is more appropriate there. There are bins numbered 0 to $\left(\mathrm{n}_{-} \mathrm{r}_{-} \mathrm{lo}_{0}-1\right)$ linearly spaced from $r=0$ to $r \_$mid. Above this, there are $\mathrm{n} \_r \_h i$ logarithmically spaced bins, with an interval r_hi_lgint.

To help bin and "unbin" the data, the following functions are provided:

function bin_of_f(f)

returns the bin number associated with an amplitude $f$. If the amplitude falls into a bin higher than $\mathrm{nf}$ then it is put into bin $\mathrm{nf}$ - this serves as a form of overflow procedure: if the highest bin contains any entries then the program should have been run with a larger number of amplitude bins. The inverse function

function f_of_bin(bin)

returns the value of the amplitude corresponding to the (logarithmic) centre of the bin (on average, the optimal value to use in reconstructing amplitudes).

For a given dipole-dipole pair, it is useful to know to which radial bins their interaction will contribute. The function

function $\operatorname{irad}(r)$

aids this by returning the bin corresponding to a given radius (the interaction region for a pair of dipoles is defined by two radii, which are converted into the range of bins which must be sampled). For each radial bin, it is useful to sample at a random radius within the bin. The random value of $r$ returned by

function rad_rnd(i)

has a probability distribution which increases linearly with $r$ within the bin $i$, replicating the distribution of radii that would be obtained by choosing a random position in the corresponding ring. Finally it can be necessary to know the lower radius of a given bin (for example when analysing binned results), which is obtained by calling 
rad_lo(i)

Given a pair of dipoles sets from evolved onia ( $\mathrm{n} 1$ dipoles in ext_dpls1 and $\mathrm{n} 2$ dipoles in ext_dpls2), the following routine

subroutine add_f_to_bins (ext_dpls1, n1, ext_dpls2, n2, fbins_single)

adds 1 to the appropriate amplitude bin of

fbins_single $(-1: b n \% n f, 0: b n \% n r)$

for a radius chosen randomly with rad_rnd in each radial bin.

\subsection{Data input and output, and initialisation}

A wide variety of information can be extracted from the evolved onium and the program needs to have the flexibility to input and output whatever information the user wants. In addition, it must be possible to restart a Monte Carlo run to add to previously determined data - this should be as automatic as possible. A number of routines are provided to make this simpler.

read_params_fdat (onm_size, maxy, n_prev_events, n_new_events)

This routine first parses the command line. Defining argn to be the $n^{\text {th }}$ command line argument, it opens arg1.dat (a file containing formatted data from the evolution) and arg1.prm (a file containing the parameters of the evolution). The number of evolutions (or events) to be performed in this run of the program (n_new_events) is the value of arg2. It then looks to see if $\arg 3$ is present: if not (or if it is a dash) then the evolution is a continuation of a previous one and the contents of arg1.prm are read in, in the following format:

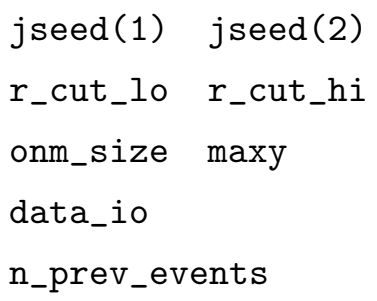

with the following definitions:

jseed (1:2) the pair of integers used as a seed for the random number generator HWRGEN, which uses l'Ecuyer's method, described in [16], as implemented in the HERWIG [17] event generator; 
r_cut_lo the lower cutoff on dipole sizes;

r_cut_hi the upper cutoff (if it is negative, then no upper cutoff is implemented);

onm_size the onium size;

maxy the maximum rapidity up to which onia are evolved;

data_io a variable provided by the user in the module data_prms holding any additional information needed by the user's program for treating the data (e.g. a range of dipole sizes being studied). It can be of any type (including user defined type);

n_prev_events the number of "events" (i.e. Monte Carlo evolutions) processed so far.

The routine also performs the initialisation which is necessary before evolution can be carried out: a call to init_lives which sets up an array tabulating dipole lifetimes as a function of their size, and a call to nullify_child_set which nullifies pointers to child dipoles (since Fortran 90 has no mechanism for specifying the initial status of a pointer). It is the user's responsibility to read in the data from unit idat (since the format and nature of the data will vary from program to program).

If arg3 is present then the file starters/arg3 (path from top directory of the distribution) is read in. The format is the same as the first three lines of arg1.prm. If either value of jseed (1:2) is zero then the default seed is used; n_prev_events is set to zero. It is then the user's responsibility to initialise any data variables and provide default values for data_io. This mechanism makes it much easier to run the same program with different values of cutoffs and rapidity (the most commonly varied parameter) by having a single set of starter files in the the starters/ directory - effectively by a command line argument, rather than by having to edit a new parameter file each time. A number of starter files are provided with the distribution and are described in the file starters/README.

write_params_fdat(onm_size, maxy, n_events, param_arg, dat_arg)

This routine is called to output the updated parameters of the evolution (usually only the seed and the number of evolutions so far, n_events, will have changed). It rewinds both the parameter and data devices. Normally the parameter and data devices (param_arg and dat_arg) will be the same as those used for input (iprm and idat) but the arguments are available (though optional) because there is occasionally a need to use different output devices (e.g. if the user wants to retain the original data). It is the user's responsibility to output the data. 
The advantage of using formatted data is that it is easy to examine or plot. But it is very inefficient for storing large amounts of data. Two routines are provided for dealing with unformatted data (the parameters file is still formatted):

read_params_ufdt (onm_size, maxy, n_prev_events, n_new_events)

write_params_ufdt(onm_size, maxy, n_events, param_dev, ufdt_dev)

They are very similar to the previous routines. The main difference is that the data device is now iufdt, which is opened for unformatted output. Since the most common use of unformatted input and output is for the binned probability distribution of the amplitude (see section 4.2) the parameter file contains the additional set of parameters: bn (a variable in the binning module, of type bin_prms) which defines the spacing of the bins. With the unformatted data files it will often be necessary to read them in again to analyse them. Because the various parameters may be needed to know how to read in and process the data a routine

read_params_old_ufdt(prnt_size, maxy, n_events)

is provided. It looks at arg1 to determine the files to use and opens them (with the OLD specifier, to prevent them being accidentally altered). It does not perform any initialisation for the evolution.

For binned amplitude data, the format is fairly constant, so routines are also provided for reading and writing the data:

subroutine allc_read_fbins (n_y, n_prev_events)

This allocates an array

fbins (-1:bn\%nf, $\left.0: b n \% n r, n_{-} y\right)$

in accord with the format for binned data discussed in section 4.2 reads it in from device iufdt. The extra dimension comes in because one might want to store results for several $\left(\mathrm{n}_{-} \mathrm{y}\right)$ values of rapidity from a single run. The reason for the argument n_prev_events is to produce the correct normalisation for the data: on disk, it is stored so that each bin contains the probability associated with it. When adding extra data, it is convenient to use a normalisation where each bin contains the number of events contributing to it. If n_prev_events is 0 then the bins are not read in, but instead initialised to zero. If the argument is not present then the normalisation of the bins is not changed.

To write the data, use the routine 
subroutine write_fbins(n_events, iufdt_arg)

It is sometimes useful to be able to specify a different device, iufdt_arg (optional), for output than for input, for example when writing out only a portion of the data. The number of events needs to be provided so that the stored normalisation on output is consistent with each bin containing a probability.

The data is stored as real rather than double precision to reduce the use of disk space.

\subsection{Some internal details}

The gluons and dipoles which are generated by evolution have to be stored, for example so that one can then work out onium-onium interactions. One of the main problems is that one doesn't know beforehand how many dipoles will be produced - the mean number of dipoles can be estimated, however the fluctuations above this are very large (see section 2.2). A solution is to dynamically allocate memory for gluons and dipoles as the evolution proceeds. This slows down the evolution (by nearly a factor of two on some systems) because of the time needed for allocation and deallocation.

The alternative is to provide fixed size arrays for the dipoles and gluons, where the size is chosen beforehand - essentially by trial and error. For cases where the largest configurations will be using a significant fraction of a machine's resources, this has the disadvantage that by having previously allocated the memory, it is taken up for the whole duration of the job (even if there is nothing stored there), whereas with dynamic allocation, the memory is used up only when it is needed.

Limits have been coded into the gluon module for the maximum number of gluons: the variable max_gluons. For the situation with dynamic allocation, this is the maximum number of gluons in each onium. It is present simply to allow the program to handle cases which might otherwise cause it to crash because they used all the available data store for that process. In the case of the fixed arrays, the quantity represents the maximum number of gluons in total (i.e. from all evolved onia). Apart from that, when using the subroutines described above for initialising onia, evolving them, etc., the functionality should not depend at all on the method of gluon storage.

There are ways round the limitations on the maximum number of dipoles. One could store very large configurations on disk (the computer would normally be doing this anyway with the swap space - but if the program is responsible then the swap space is still left for other processes). Alternatively, there are situations where the dipole structure need not be stored at all. The occasions when a stored dipole structure is needed is when looking at some kind of correlation between dipole pairs (such as onium-onium interaction), because for each dipole, 
one needs access to all the other dipoles. But in these situations, the time per configuration is normally the limiting factor, since this is proportional to the square of the number of dipoles. In cases where the time taken for some analysis is linear in the number of dipoles, often, it is not necessary to have knowledge of the whole configuration. So an evolution routine could be written which called an analysis routine each time it reached the "tip" of a "branch" of a dipole "tree", and it would never have to store more than a single path from the base of the tree to the furthest "tip".

\subsection{Compilation}

The program is provided in the form of a number of separate files, the contents of which are documented in README files. To aid in the compilation process, two shell scripts are provided which select the correct set of source files. Detailed information on their functioning is provided as part of the distribution, however a summary is given here:

mkf driver [ dyn | sml | lrg ]

This is for compiling a driver routine which uses formatted data i/o. The name of the driver should be provided without the ff90 extension. The second command line argument indicates the kind of gluon and dipole storage used - the default is to use dynamic allocation (corresponding to the option dyn). Two fixed size array storage options are also provided: sml (up to 5000 gluons) and Irg (up to 400000 gluons, which with a lower cutoff of $0.01 b$, should be adequate for evolution up to rapidities of $y \simeq 15)$. The executable is named driver_dyn (or sml or lrg as appropriate) to allow multiple copies of the executable, with different gluon storage, to coexist.

mku driver [ dyn | sml | lrg | noev ]

This is for compilation of drivers which use unformatted data i/o. It also compiles in the routines and modules associated with binning of the amplitude. The command line arguments are the same as for mkf, except for the extra option available as the second argument: noev. This is to be used for drivers which analyse data from a previous evolution - no evolution routines are compiled, and the data and parameter files are opened with the 'OLD' specifier, meaning that they cannot be modified. Naming of the executable follows the same convention as for mkf except when the noev option is used, in which case the executable is named driver (since there should be no need to have multiple copies compiled with different gluon storage methods). 


\subsection{Test run}

The run described here tests the evolution of the onia, determination and binning of the interaction amplitude, and subsequent analysis of data for onium-onium interactions. It is for moderate rapidities and very small statistics to ensure that it runs quickly (a few seconds) on most systems. It is necessary to first compile the evolution routine (to be found in the sample/ directory of the distribution) with

$>$ mku onm-onm-bnd dyn

which will include in dynamic allocation of memory for gluons and dipoles. The first command starts an evolution (with 10 events) where the maximum rapidity for each onium is 4 , giving a total rapidity for the collisions of 8 . The lower cutoff used is 0.1 times the onium size. The binned, unformatted data are stored in test_y8.ufdt (beware: this file is over 1MB in size) and the parameters in test_y8.prm. The format of the variable data_io (together with the defaults used here) is

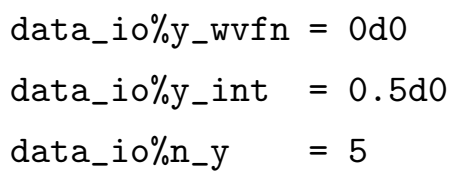

which specifies that the onium-onium interaction is determined for 5 values of rapidity, each separated by 0.5 (note that this is for each onium - so the intervals in total rapidity are 1.0). The maximum rapidity at which dipoles are extracted from each onium is maxy, but each onium is evolved to data_io\%y_wvfn (or maxy, if this is larger) allowing different runs to effectively use the same dipole configurations but to examine the interactions at different values of rapidity. Various messages are output at the start of the program, as different parts of the startup procedure are accomplished. The line dealing with backups may be preceded by other messages on some systems (e.g. those using the NAG compiler — see section 4.8), because the compiler does not offer the facility of running a system command.

The next command causes the evolution to be continued for a further 5 "events" (simply to allow a test of the continuation function). One then wants to analyse the results of the evolution. In this test we will look at the total amplitudes (the onium-onium amplitude integrated over all impact parameters, which is equal to half the onium-onium total crosssection). The program to do this is compiled with

mku ftot noev 
The option noev ensures that unnecessary routines (such as those required for evolution) are not compiled in. The output includes various messages at the beginning indicating the initialisation procedure (these messages are sent to standard error, while the numerical output is sent to standard output, to allow it to be redirected to a file). The format of the numerical output from noev is one line for each value of rapidity, where each line has:

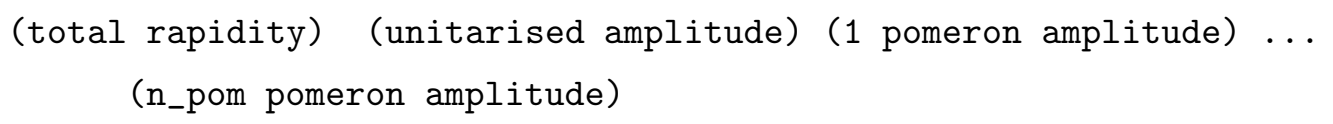

(all on one line), and n_pom $=4$ is a parameter in the program. It is the absolute values of the amplitudes which are output.

A second test run, which tests the formatted data output is provided and documented within the distribution. In addition, some other driver and analysis routines are provided. These can all be found in the samples/ directory.

Examples of results produced with OEDIPUS can be found in [13, 14].

Typical rapidities for which adequate statistics are accessible with a day's running are $Y \leq 20$, using a lower cutoff of $0.01 b$.

\subsection{Distribution}

The distribution (provided as a compressed tarred file) consists of a number of directories. Details of their contents are documented in extensive README files. A shell script is provided to help automate the installation procedure. Instructions are also included for installation by hand.

\subsection{Portability}

The main issues of portability relate to accessing command line arguments and causing a shell script (which performs backups of data and parameter files) to be executed. Interfaces to the system routines to perform these functions are provided as routines whose names start with

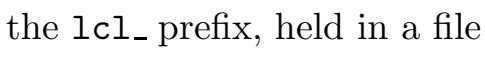

basic_src/common/lcl_???.f90

where ??? may be dec, nag or std (or the user can generate a new file for his/her operating system). The file to be used must be specified as part of the installation procedure. With the NAG compiler (version 2.1), there does not seem to be any way of causing a shell script to be executed (the usual unix system subroutine seems to be absent). The routines for accessing 
command line arguments while available, do not seem to be documented. If the Fortran 90 compiler offers no mechanism for accessing the command line arguments then one can use lcl_std.f90, which prompts the user for the information held in each of the arguments. It conforms to the standard.

Local implementations are also necessary for writing large, unformatted arrays to disk (on some systems, the i/o buffer is not sufficiently large and the array must be written in small sections). Details are provided in the 1c1_???.f90 files.

To port to a non unix system (such as VMS), it would in addition be necessary to rewrite the compilation scripts.

\subsection{Global data}

All global data is stored in the form of modules. These are summarised in table 1 (numbers in brackets refer to default values).

In addition there are modules associated with the binning routines. These are summarised in table 2.

\section{Acknowledgements}

I would like to thanks B.R. Webber and A.H. Mueller for suggesting this work, as well as for many useful comments. In addition, M.H. Seymour's introduction to Monte Carlo simulation techniques (in 18]) has been very helpful during the development of this program. 


\begin{tabular}{|c|c|c|}
\hline Module name & Variables & Description \\
\hline \multirow[t]{3}{*}{ constants } & alpha_s & $\alpha_{S}$, the strong coupling constant $(0.17777 \ldots)$ \\
\hline & nc & $N_{C}$, the number of colours in QCD (3) \\
\hline & alpha_no_nc & $\begin{array}{l}\text { the value of } \alpha_{S} \text { to be used when it appears without a } \\
\text { factor of } N_{C} \text { (=alpha_s). }\end{array}$ \\
\hline \multirow[t]{3}{*}{ cuts } & r_cut_lo & the lower cutoff \\
\hline & r_cut_hi & the upper cutoff \\
\hline & hi_cut_implem & true if an upper cutoff is being used \\
\hline \multirow[t]{3}{*}{ iomodule } & iprm & i/o device for parameters (10) \\
\hline & idat & i/o device for formatted output (12) \\
\hline & iufdt & i/o device for unformatted data (14) \\
\hline \multirow[t]{4}{*}{ gluons } & type gluon & the type definition for gluons \\
\hline & type dipole & the type definition for dipoles \\
\hline & type dpl_pntr & type definition for a pointer to a dipole \\
\hline & max_gluons & the maximum number of gluons to be generated \\
\hline data_defs & data_io & $\begin{array}{l}\text { variable of user chosen type, with details of data i/o. } \\
\text { This module must be provided by the user. }\end{array}$ \\
\hline sub_defs & & interface blocks for all the main subroutines \\
\hline
\end{tabular}

Table 1: A list of the modules (and their main contents) needed by user's programs

\begin{tabular}{|l|l|l|}
\hline binning & type bin_prms & $\begin{array}{l}\text { type definition to store all the parameters for binning } \\
\text { of amplitudes }\end{array}$ \\
\cline { 2 - 3 } & bn & variable actually containing the information \\
\hline fbins_mdl & fbins & array containing amplitude bins \\
\hline bin_interface & & interface blocks for the binning routines \\
\hline
\end{tabular}

Table 2: Modules associated with routines for binning the amplitude probability distribution 


\section{References}

[1] Y. Y. Balitskiǐ and L. N. Lipatov, Sov. Phys. JETP 28 (1978) 822.

[2] E. A. Kuraev, L. N. Lipatov, and V. S. Fadin, Sov. Phys. JETP 45 (1977) 199.

[3] L. N. Lipatov, Sov. Phys. JETP 63 (1986) 904.

[4] J. Bartels, H. Lotter, and M. Wüsthoff, Zeit. f. Phys. C68 (1995) 121.

[5] A. Bialas and R. Peschanski, hep-ph/9512427.

[6] J. Bartels, J. R. Forshaw, H. Lotter, and M. Wüsthof, DESY preprint 95-253, hep$\mathrm{ph} / 9601201$.

[7] A. H. Mueller, Nucl. Phys. B415 (1994) 373.

[8] A. H. Mueller and B. Patel, Nucl. Phys. B425 (1994) 471.

[9] A. H. Mueller, Nucl. Phys. B437 (1995) 107.

[10] Z. Chen and A. H. Mueller, Nucl. Phys. 451 (1995) 579.

[11] N. N. Nikolaev and B. G. Zakharov, Zeit. f. Phys. C64 (1994) 631.

[12] N. N. Nikolaev, B. G. Zakharov, and V. R. Zoller, JETP Lett. 59 (1994) 6.

[13] G. P. Salam, Nucl. Phys. B449 (1995) 589.

[14] G. P. Salam, Cavendish-HEP-95/05, hep-ph/9509353, to appear in Nucl. Phys. B (1996).

[15] A. H. Mueller and G. P. Salam, Cavendish-HEP-95/06, in preparation.

[16] F. James, Comp. Phys. Comm. 60 (1990) 329.

[17] G. Marchesini et al., Comp. Phys. Comm. 67 (1992) 465.

[18] M. H. Seymour, Predictions for Higgs and Electroweak Boson Production, PhD thesis, Cambridge University, 1992. 


\section{TEST RUN}

The input and output of the test run are shown below:

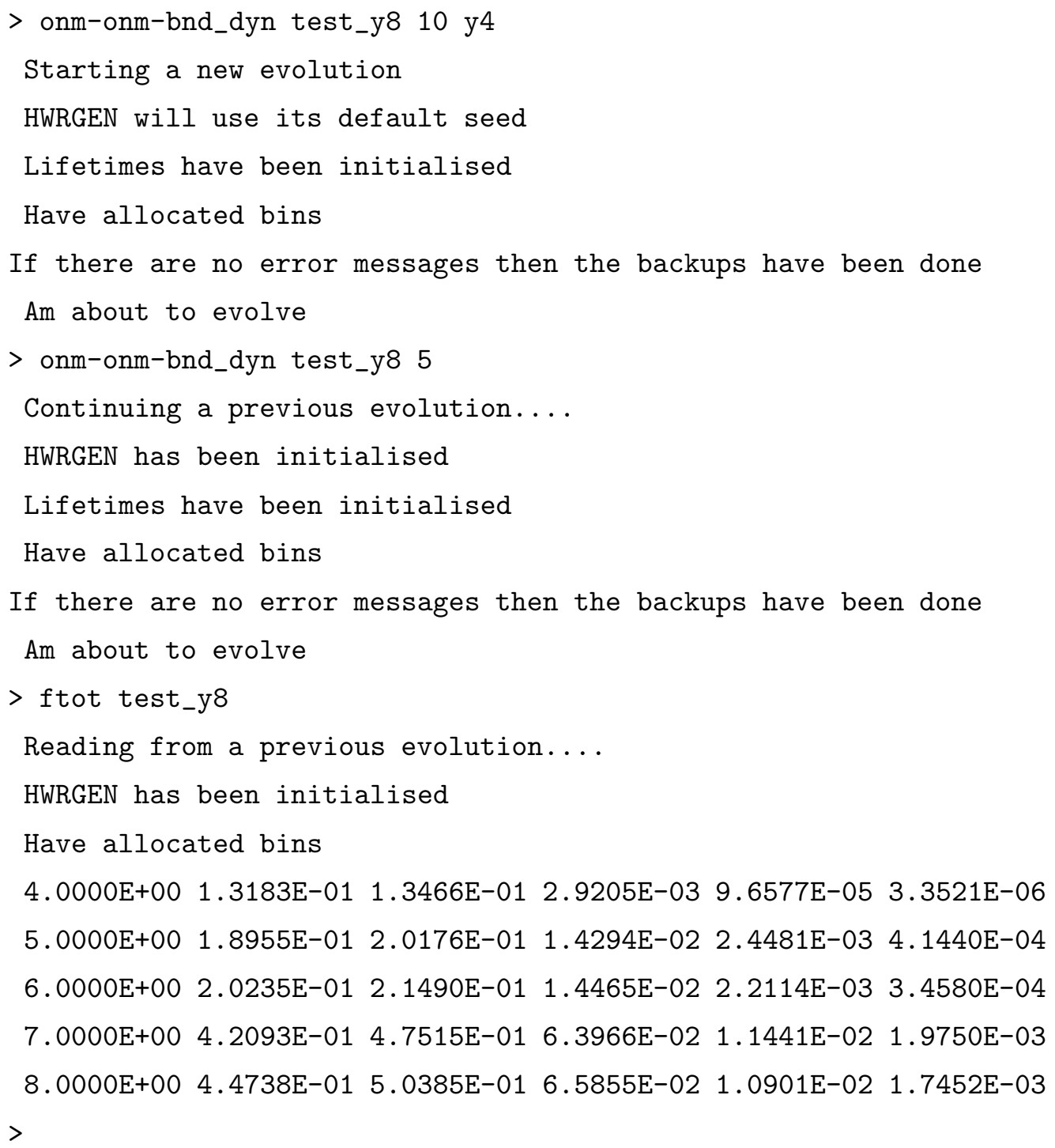

The starter routine $\mathrm{y} 4$ is the following (default seed, lower cutoff of 0.1 , no upper cutoff $(-2.0)$, onium size of 1.0 , and maximum rapidity for each onium of 4.0$)$ :
$0 \quad 0$
$0.1-2.0$
$1.0 \quad 4.0$ 